\title{
Turbulent and Chaotic Dynamics Underlying Solar Magnetic Variability
}

Article in The Astrophysical Journal · November 1995

DOI: $10.1086 / 176583$

CITATIONS

51

3 authors:

\section{John K. Lawrence}

California State University, Northridge

147 PUBLICATIONS 1,091 CITATIONS

SEE PROFILE

\section{Alexander Ruzmaikin}

California Institute of Technology

400 PUBLICATIONS $\mathbf{5 , 0 8 0}$ CITATIONS

SEE PROFILE
READS

19

\section{Ana Cadavid}

California State University, Northridge

98 PUBLICATIONS 878 CITATIONS

SEE PROFILE

All content following this page was uploaded by Ana Cadavid on 26 September 2014. 
THE ASTROPHYSICAL JoURNAL, 455:366-375, 1995 December 10

(C) 1995. The American Astronomical Society. All rights reserved. Printed in U.S.A.

\title{
TURBULENT AND CHAOTIC DYNAMICS UNDERLYING SOLAR MAGNETIC VARIABILITY
}

\author{
J. K. Lawrence, A. C. Cadavid, AND A. A. Ruzmaikin \\ San Fernando Observatory, Department of Physics and Astronomy, California State University, Northridge, Northridge, CA 91330-8268 \\ Received 1995 March 13; accepted 1995 June 15
}

\begin{abstract}
We examine the temporal scaling properties of solar magnetic activity on timescales from days to decades. Because of more than 63,000 usable data points, we concentrate on the daily International Sunspot Number. Some results have been checked with other data sets, primarily the $10.7 \mathrm{~cm}$ microwave flux with about 16,000 data points. Such time series provide a measure whose scaling and intermittency properties are analyzed.

By means of correlation analysis and both Fourier and wavelet spectral analysis, we distinguish two regimes of temporal behavior of the magnetic variability. The scaling of the time series is analyzed in terms of multiplicative cascade processes which prove to be invariant over more than two decades of scale from about $2 \mathrm{yr}$ down to about 2 days or less. We interpret this result to indicate generic turbulent structuring of the magnetic fields as they rise through the convection zone. We find that a low-dimensional, chaotic behavior in the sunspot number operates entirely at timescales longer than a transition threshold scale of about $8 \mathrm{yr}$. Magnetic variability on timescales between $2 \mathrm{yr}$ and $8 \mathrm{yr}$ apparently requires handling by direct simulation.
\end{abstract}

Subject headings: Sun: activity - Sun: magnetic fields - sunspots - turbulence

\section{INTRODUCTION}

The highly variable magnetic fields appearing on the surface of the Sun are important both in their effects and in their causes. On the one hand, these fields modulate not only solar particle flows and cosmic rays striking Earth's magnetosphere but also the solar irradiance at Earth, with potentially crucial effects on terrestrial climate. On the other hand, the spatial and temporal patterns of the emerging flux are the best clues available to the nature of plasma motions in the convection zone and to the dynamo operating therein. In previous works (Lawrence, Ruzmaikin, \& Cadavid 1993; Cadavid et al. 1994; Lawrence, Cadavid, \& Ruzmaikin 1994, 1995), we have made use of multifractal analysis to show that the spatial distributions of small-scale photospheric magnetic fields have selfsimilar scaling and to argue that this may display a generic turbulent structure (Lawrence et al. 1995). We wish to apply this approach, along with other modern methods of time series analysis, to see what new information may be extracted from temporal indices of the global solar magnetic variability. Here the Sun is viewed as a star, and our techniques can be applied to other stars when sufficiently long data time series have been accumulated.

The daily International (formerly Zürich) Sunspot Number series has been investigated extensively over the years. Nevertheless, new information continues to be wrung from it as new methods are applied. The sunspot number is only indirectly related to the actual physics of the photosphere, but it does provide a quantitative indication of the level of solar activity. It also has the unique advantage of spanning nearly two centuries of time (nearly three centuries in the case of the yearly average numbers). This has arisen from the simplicity of definition of the sunspot number by Johann Rudolf Wolf in such a way that its value can be reconstructed from earlier observations as far back as A.D. 1700. Modern measures of activity necessarily cover much shorter time spans. Among other data sets, however, we have used the daily Ottawa $10.7 \mathrm{~cm}$ microwave flux measurements $\left(F_{10.7}\right)$, obtained from NOAA's National Data Center, and covering over $40 \mathrm{yr}$, to compare some of our results.
The idea has been abroad for many years that the quasiperiodic $11 \mathrm{yr}$ sunspot cycle, variations in amplitude of sunspot maxima, sporadic Maunder-like minima, and the like, reflect the effect of a low-dimensional, chaotic dynamical system (Ruzmaikin 1981). These have been modeled by systems of a small number of coupled, nonlinear differential equations (Ruzmaikin 1981; Weiss, Cattaneo, \& Jones 1984). A study by Mundt, Maguire, \& Chase (1991) has indicated that within the sunspot number series lies evidence of a global chaotic dynamical system with low-dimensional $(D \approx 2.3)$ attractor. We employ a variation on the method of Grassberger \& Procaccia (1983a) to find that the dynamical system in question operates entirely at timescales $\gtrsim 8 \mathrm{yr}$. This leaves open the question of the short-term fluctuations of the sunspot number, and our approach to these is based on their simultaneous intermittence and scale invariance properties.

Correlation and spectral analyses of the sunspot number reveal strong signals based on the 27 day solar rotation period and the $11.2 \mathrm{yr}$ activity cycle. They also reveal a drop in the general level of correlation of the series for timescales longer than about a year. More significantly for our purposes, Fourier analysis of the daily sunspot number indicates self-similar, power-law scaling in the range between the rotation period and about a year.

A technique which is finding diverse areas of application and which is well suited to the study of scale invariance is wavelet analysis (for reviews, see Meneveau 1991 and Farge 1992). A scaling, or "zoom lens," parameter in the wavelet transform of a function can reveal the location and strength in a given time series of structures of different durations. Similarly, a Haar wavelet transform allows us to examine the flatness of the distribution of sunspot number increments as a function of the temporal resolution at which they are observed. This indicates that the time series is of intermittent, rather than Gaussian, structure on timescales below about a year. A complex Morlet wavelet transform allows a direct display of the structural properties of the montly and yearly average sunspot number time series. Below a timescale of 1 or $2 \mathrm{yr}$ we find intermittent structure. The $11.2 \mathrm{yr}$ cycle is very prominent. At longer time- 
scales we find structures at scales of $\sim 22 \mathrm{yr}$ and $\sim 45 \mathrm{yr}$ as well as a "Gleissberg structure" at $\sim 90 \mathrm{yr}$ in good agreement with the result of Feynman \& Gabriel (1990).

The daily sunspot number and the $F_{10.7}$ time series provide mathematical measures whose scaling and intermittency (e.g., multifractal) properties we analyze in terms of a random multiplicative cascade process (Chhabra \& Sreenivasan 1991a, b, 1992; Lawrence, Cadavid, \& Ruzmaikin 1995). Here the measure in an arbitrary time interval is pictured as being divided between its two halves in the fractions $M$ and $1-M$, where $0 \leq M \leq 1$ is drawn randomly from a scale-invariant probability distribution. This distribution can be determined by direct sampling of the time series. What we find is akin to a wavelet power spectrum whose output is a function at each scale rather than a single number.

After allowing for the effects of solar rotation and the sunspot cycle, we find the multiplier probability distribution density to be invariant over more than two decades of scale from about 2 days to about 2 yr. The scale invariance and the form of the invariant probability distribution we find is characteristic of a wide range of turbulent phenomena (Chhabra \& Sreenivasan 1991a, b; 1992; Lawrence et al. 1995). This is our principal result; we interpret it to indicate generic turbulent structuring of solar fields as they rise through the convection zone.

In $\S 2$ we describe the International Sunspot Number and other temporal indices of solar activity used in this work. Section 3 includes descriptions of several kinds of analysis of the data: the correlation and mutual information functions, Fourier and wavelet spectral analysis, random cascade models, and the transition to the behavior of a low-dimensional dynamical system at long timescales. Results are discussed and conclusions presented in $\S 4$.

\section{OBSERVATIONAL DATA}

The analyses we propose require data covering the widest possible range of timescales. They must span as long a time as possible without interruption, with the finest possible temporal resolution. The best available data set, according to these criteria, is the daily sunspot number $R(t)$. This measure of solar activity was introduced in 1848 by Wolf (Bray \& Loughhead 1964, p. 237) and defined to be the total number of spots visible on the solar disk plus 10 times the number of sunspot regions. These are multiplied by a " personal factor" intended to reconcile observations made under different circumstances. Daily observations of $R(t)$ accurate to a few percent are available from 1849. By compiling earlier observations from a variety of sources, Wolf was able to reconstruct the daily sunspot number, with a few modest gaps, and with reasonable accuracy, back to 1818 . This gives a total of about 63,000 useful data points covering scales from a day to many decades, and our results are based on these data. Also reconstructed were monthly average sunspot numbers back to 1749 and yearly averages back to 1700 , although these values may be inaccurate by tens of percents.

The lower panel of Figure 1 shows the sunspot number for 1986 through 1989, including part of Cycle 22 and the preceding minimum. This span is chosen to display phenomena over that range of timescales with which we are principally concerned in this paper: from a few days to a few years. It also compares phenomena at sunspot minimum to those at sunspot maximum.

A secondary data set is the $10.7 \mathrm{~cm}(2800 \mathrm{MHz})$ microwave

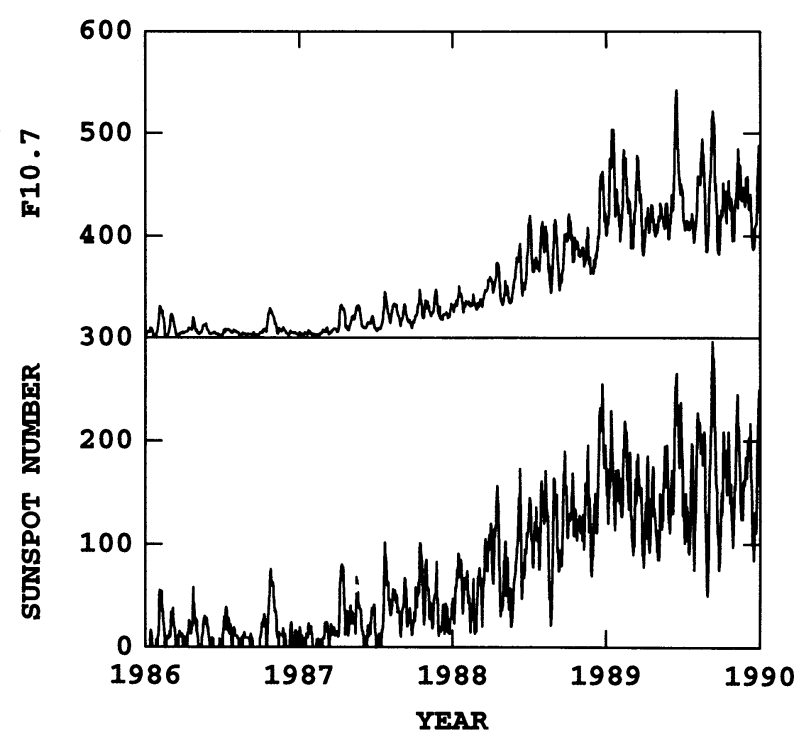

FIG. 1.-Daily International Sunspot Number (lower) and $10.7 \mathrm{~cm}$ microwave flux (upper) from 1986 January 1 to 1990 January 1 . The minimum value of $F_{10.7}$ has been set to a value of 300 arbitrary units to separate the plots.

flux measurements $\left(F_{10.7}\right)$ recorded daily near Ottawa and Penticton, Canada, since 1947 February 14. There are about 16,000 data points. This signal originates in the upper chromosphere and lower corona and appears to comprise three main components. First is a background emission, which we subtract from the data so that the minimum value of $F_{10.7}$ is set to zero. Second, transient bursts from solar flares are corrected for in the data published in NOAA's Solar Geophysical Data Bulletin. The third component, which roughly tracks the number of active regions present on the solar disk, is what we analyze here. The upper panel of Figure 1 shows a plot of these data also from 1986 through 1989.

Other data sets considered are as follows. Daily calcium emission indices, in active region plages between 1970 and 1987 , provide a data string of about 6000 points. This is similar in length to the Stanford mean magnetic field observations between 1975 and 1991. These data were acquired from NOAA's World Data Center. The ERB/Nimbus 7 (Hoyt et al. 1992) spacecraft observations of solar irradiance fluctuations provide about 5000 days of mostly uninterrupted data between 1978 and 1991. The daily Photometric Sunspot Index (Willson et al. 1981), estimating the sunspot contribution to irradiance shifts, has been calculated for the same period.

\section{ANALYSIS OF THE SUNSPOT TIME SERIES}

\subsection{Correlation Function and Mutual Information}

Characteristic timescales in temporal data are of central importance. For example, these indicate important periodicities, such as the 27 day solar rotation period and the $11.2 \mathrm{yr}$ sunspot cycle. In addition, this analysis will be used to choose a time lag for use in searching for a low-dimensional attractor in the longer term solar variability. A standard tool for finding characteristic timescales in temporal data is the correlation function $C(\tau)$ of the time series. We take the value of the series $R(t)$ at two times separated by an interval $\tau$, subtract the mean value of the series from each, multiply, and average over the series, which is presumed to be stationary (see below):

$$
C(\tau)=\langle[R(t+\tau)-\langle R\rangle][R(t)-\langle R\rangle]\rangle .
$$


The correlation function for the daily sunspot number is shown as the dotted line in Figure 2. It shows several peaks representing the $\sim 27$ day solar rotation period and subharmonics. The correlation function passes through zero at about $2^{10}$ days $\approx 3 \mathrm{yr}$, indicates maximum anticorrelation at about $2^{11}$ days $\approx 5.5 \mathrm{yr}$, and then a correlation peak signifying the 11.2 yr sunspot cycle. Subsequent maxima and minima display subharmonics of the sunspot cycle. These decrease in amplitude and then increase again near $\sim 90 \mathrm{yr}$, indicating the Gleissberg cycle in the sunspot maxima. The correlation function suggests that the sunspot numbers first become completely decorrelated at its first zero crossing at about $3 \mathrm{yr}$. This zero crossing is somewhat arbitrary, however, because equation (3.1) involves subtracting the average of a time series with all positive values.

An alternative measure of correlation, which avoids this arbitrariness, is the "mutual information" $I(\tau)$ (Gallager 1968; Abarbanel et al. 1993). To calculate this we find the joint probability $p\left(R_{i}, R_{j}, \tau\right)$ of finding a value $R_{i}$ in the series and a value $R_{j}$ a time $\tau$ later. This can be evaluated directly from the data. If the measurements are uncorrelated then $p\left(R_{i}, R_{j}, \tau\right)=$ $p\left(R_{i}\right) p\left(R_{j}\right)$, the product of the individual probabilities. Thus we divide the joint probability by the uncorrelated probability, take the logarithm (to base 2) to get zero for no correlation, and average over the ensemble of joint measurements:

$$
I(\tau)=\sum_{i, j} p\left(R_{i}, R_{j}, \tau\right) \log _{2}\left[\frac{p\left(R_{i}, R_{j}, \tau\right)}{p\left(R_{i}\right) p\left(R_{j}\right)}\right] .
$$

Because this has the form of the negative of an entropy, it is called the mutual information of the time series. It indicates the amount of information gained about the value of a number in the series by knowing the value a time $\tau$ earlier. The mutual information for the daily sunspot number is shown as the solid line in Figure 2. We can see the signal of the solar rotation at $\tau \approx 27$ days, together with several of its harmonics. The $11.2 \mathrm{yr}$ periodicity and harmonics are apparent as is a peak near $90 \mathrm{yr}$

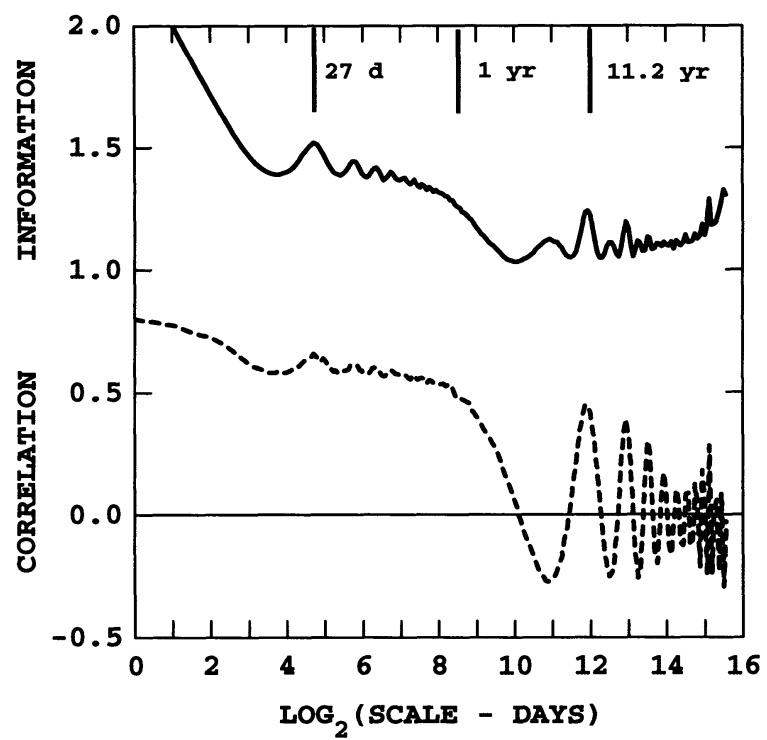

FIG. 2.-Mutual information (solid line) and correlation function (dashed line) for the daily sunspot number vs. timescale in days. The mutual information has been displaced upward for clarity. The timescale starts at 1 day with each unit step along the abscissa representing a doubling of scale. Also indicated for reference are three timescales: the 27 day solar equatorial rotation period, the terrestrial year, and the 11.2 yr mean sunspot cycle. for the Gleissberg cycle. Anticorrelation minima in the correlation function show here as maxima, indicating that knowledge of a current sunspot maximum or minimum increases our information about the subsequent minima or maxima. The overall minimum of the mutual information does come at $\tau \approx 3$ yr. Knowledge of the sunspot number at a given time gives little information about what number to expect 3 yr later. Below we will use the first local minimum of the mutual information at $\tau \approx 13$ days as the time lag to use for finding lowdimensional attractors in the series.

Most importantly for our analysis, both the correlation function and the mutual information indicate a relatively high correlation of the sunspot numbers at short timescales, followed by a steep dropoff between 1 and $2 \mathrm{yr}$.

\subsection{Spectral Analysis}

\subsubsection{Fourier Spectrum and Stationarity of the Sunspot Number Series}

Figure 3 shows the Fourier power spectrum of the daily sunspot number series, plotted versus period rather than frequency. This is an average taken from separate analyses of seven nonoverlapping data segments of length $2^{13}$ days $\approx 22$ yr each. A Welch windowing function was applied. A longperiod peak in the figure apparently represents the $11.2 \mathrm{yr}$ sunspot cycle. The last point corresponds to the full $22 \mathrm{yr}$ period, and its value may be a finite length effect. A distinct peak with period $\sim 27$ days represents the rotation of the Sun as viewed from Earth, coupled with an inhomogeneous distribution of magnetic activity in solar longitude. Between these two peaks the spectrum displays a power-law dependence on scale (and hence on frequency). The spectral index is $\beta=0.66 \pm 0.04$, in agreement with Morfill et al. (1991). Stochastic time series with spectral index $\beta<1$ are stationary, that is, their statistical properties are time independent. Most of our analysis depends on the stationarity of the time series.

\subsubsection{Wavelet Analysis}

The wavelet transform (for reviews, see Meneveau 1991 and Farge 1992) of a function $R(t)$ is its convolution with a set of

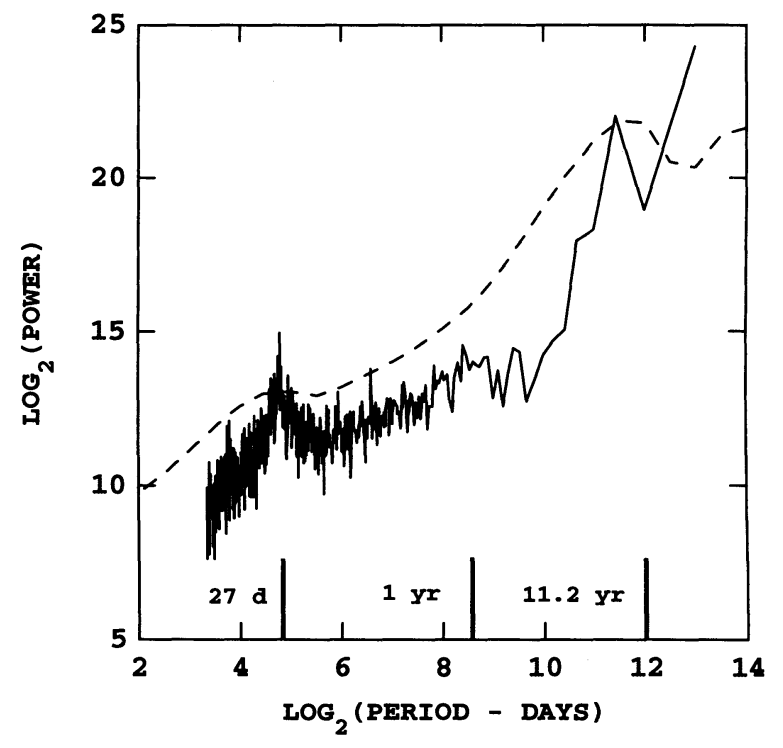

FIG. 3.-Fourier power spectrum of the daily sunspot number (solid line) vs. period in days; corresponding Haar wavelet power spectrum (dashed line). These are plotted vs. timescale or period rather than frequency. This changes the sign of the slopes. Also indicated for reference are the 27 day solar equatorial rotation period, the terrestrial year, and the $11.2 \mathrm{yr}$ mean sunspot cycle. 
basis functions that are dilations and translations of a single "mother function" $\Psi$ :

$$
W(a, b)=\frac{1}{\sqrt{a}} \int d t R(t) \Psi\left(\frac{t-b}{a}\right) .
$$

The parameter $a$ governs the dilations of $\Psi$, and $b$ its translations. To be acceptable, the mother function must give a zero response to a uniform function, so it must contain both positive and negative parts. Such a mother function is designed to produce a strong response at point $b$ to structure of scale $a$ at time $t$, but weak or no response otherwise. The wavelet transform in $d$ dimensions (here $d=1$ ) is a function of $d+1$ variables. The extra variable $a$ serves as a kind of zoom lens, and by adjusting it we can study the structure of $R(t)$ at different levels of temporal coarseness.

The simplest real mother function is the Haar wavelet: $\Psi_{\mathrm{H}}(t)=-1$ on the interval $-1 \leq t<0, \Psi_{\mathrm{H}}(t)=+1$ on the interval $0<t<1$, and 0 otherwise. Transforms with respect to real wavelets mix information about the signal being analyzed with the structure of the mother function. For example, the Haar wavelet computes the difference between the total measures contained in two equally long, adjacent intervals of the series, and it thus responds to the first derivative of a continuous signal, or to the increments of a stochastic time series. In what follows, we will look at variants of this procedure. Of special interest will be the ratio of the measure in each of the two intervals, rather than their difference. The statistics of this quantity describe the multiplicative cascade process to be used below in multifractal analysis of the time series.

By integrating $W(a, b)$ over $b$ and squaring, we can generate a "wavelet power spectrum" as shown in Figure 3 for the Haar wavelet. This is just the Fourier power spectrum smoothed by the Fourier transform of the wavelet basis function at each scale. In this case the 27 day and 11.2 yr signals are evident, but they are sufficiently broadened that we cannot determine a spectral index for the scaling region between them.

Wavelet analysis can be used to examine the scaling behavior of a variety of numerical properties of a time series. Of some interest is the "flatness" of the probability distribution of the series, a diagnostic for intermittence. This is defined at temporal resolution $a$, for a stationary series, as

$$
\Phi(a) \equiv \frac{\left\langle W^{4}(a, b)\right\rangle_{b}}{\left\langle W^{2}(a, b)\right\rangle_{b}^{2}} .
$$

If the series has a Gaussian probability density, we will find $\Phi=3$. An intermittent series, with long tails in its distribution, gives $\Phi>3$. To study the dependence of $\Phi$ on temporal resolution, we construct the Haar wavelet transform, calculate $W^{2}(a, b)$ and $W^{4}(a, b)$ from its components for all $a$ and $b$, and perform the time averages over $b$ to find the flatness for different scales $a$. Because we are using the Haar wavelet we see the flatness of the increments of the coarsened series. Figure 4 shows the resulting "flatness spectrum" $\Phi(a)$ for both the daily and monthly sunspot numbers. For $a \lesssim 1 \mathrm{yr}$. we find $\Phi \approx 6-7$ indicating non-Gaussian intermittence; for $a \gtrsim 1 \mathrm{yr} \Phi$ drops to about 3 indicating a more nearly Gaussian distribution. Thus the behavior of the sunspot time series divides into two temporal regimes. On scales shorter than a year or two the behavior is intermittent; on longer timescales it is more regular. We note that intermittence is characteristic of turbulent phenomena.

We can obtain a visual impression of the two behaviors by a different wavelet analysis. A complex mother function, such as

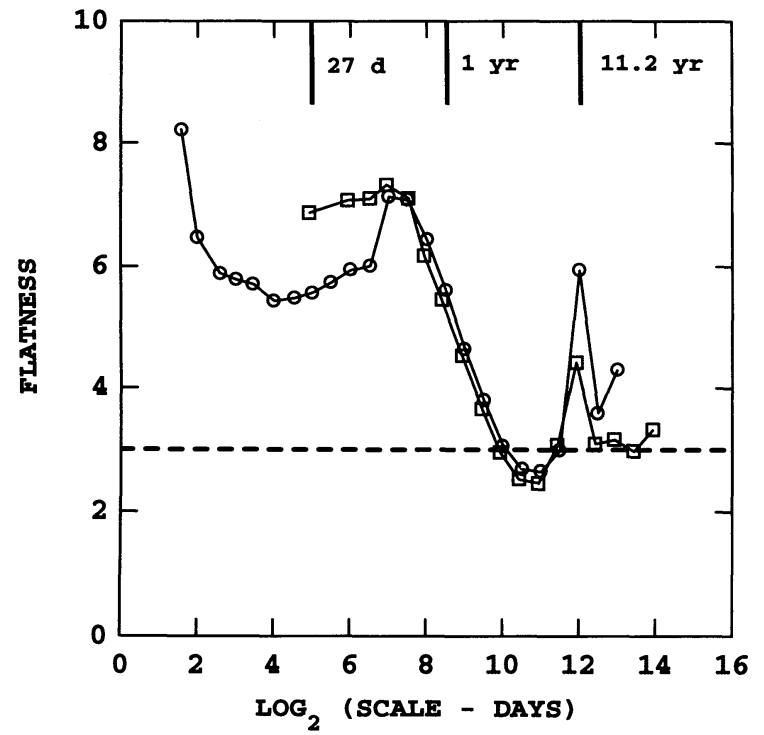

Fig. 4.-Flatness spectrum of daily sunspot number (circles) and monthly sunspot number (squares) vs. timescale. The dashed line denotes flatness 3 , which is characteristic of Gaussian noise. Also indicated for reference are the 27 day solar equatorial rotation period, the terrestrial year, and the $11.2 \mathrm{yr}$ mean sunspot cycle.

the Morlet wavelet, responds directly to the amplitudes of activity structures in the data at different timescales (Meneveau 1991; Farge 1992). The Morlet mother function is

$$
\Psi_{M}(k, t)=\pi^{-1 / 4}\left(e^{-i k t}-e^{-k^{2} / 2}\right) e^{-t^{2} / 2} .
$$

For $k>5$ the second term is negligible.

Figure 5 shows the modulus $\left(W^{*} W\right)^{1 / 2}$ of the Morlet wavelet transform of the monthly sunspot number over the period from 1950 to 1990 . The horizontal position of features in this plot gives their location in time. The vertical position indicates their temporal scale, the parameter $a$ in equation (3.3). The top horizontal row represents temporal features on the scale of 1 month $\approx 2^{5}$ days. The timescale of the features is doubled for each unit step downward on the plot. Horizontal lines are added for reference at the timescales of $1 \mathrm{yr} \approx 2^{8.5}$ days and $11.2 \mathrm{yr} \approx 2^{12}$ days. A cascade of short-timescale features at the top of the image proceeds to a smaller number of longer timscale features as we move downward to the scale of a year or two. This represents the short-time regime of solar activity fluctuations. The feature spanning the image horizontally with scale between $2^{11}$ and $2^{12}$ days indicates the sunspot cycle and the onset of the long-time regime of activity fluctuations.

Figure 6 similarly displays the modulus of the Morlet wavelet transform of the annual sunspot number from 1700 to the present. Data earlier than 1818 should be regarded with caution. The top horizontal row now represents temporal features in the time series on the scale of $1 \mathrm{yr} \approx 2^{8.5}$ days, and each unit step downward again corresponds to a doubling of the timescale being examined. A horizontal line has been added for reference at a timescale of $11.2 \mathrm{yr} \approx 2^{12}$ days. Once again the feature spanning the image at scales between $2^{11}$ and $2^{12}$ days represents the sunspot cycle. This feature is highly structured, indicating that the sunspot cycle is far from uniform in nature. At times, as in recent decades, the cycle is strong. At other times, such as early in the 19 th century, the cycle is much weaker. Changes in vertical position of the feature indicate 


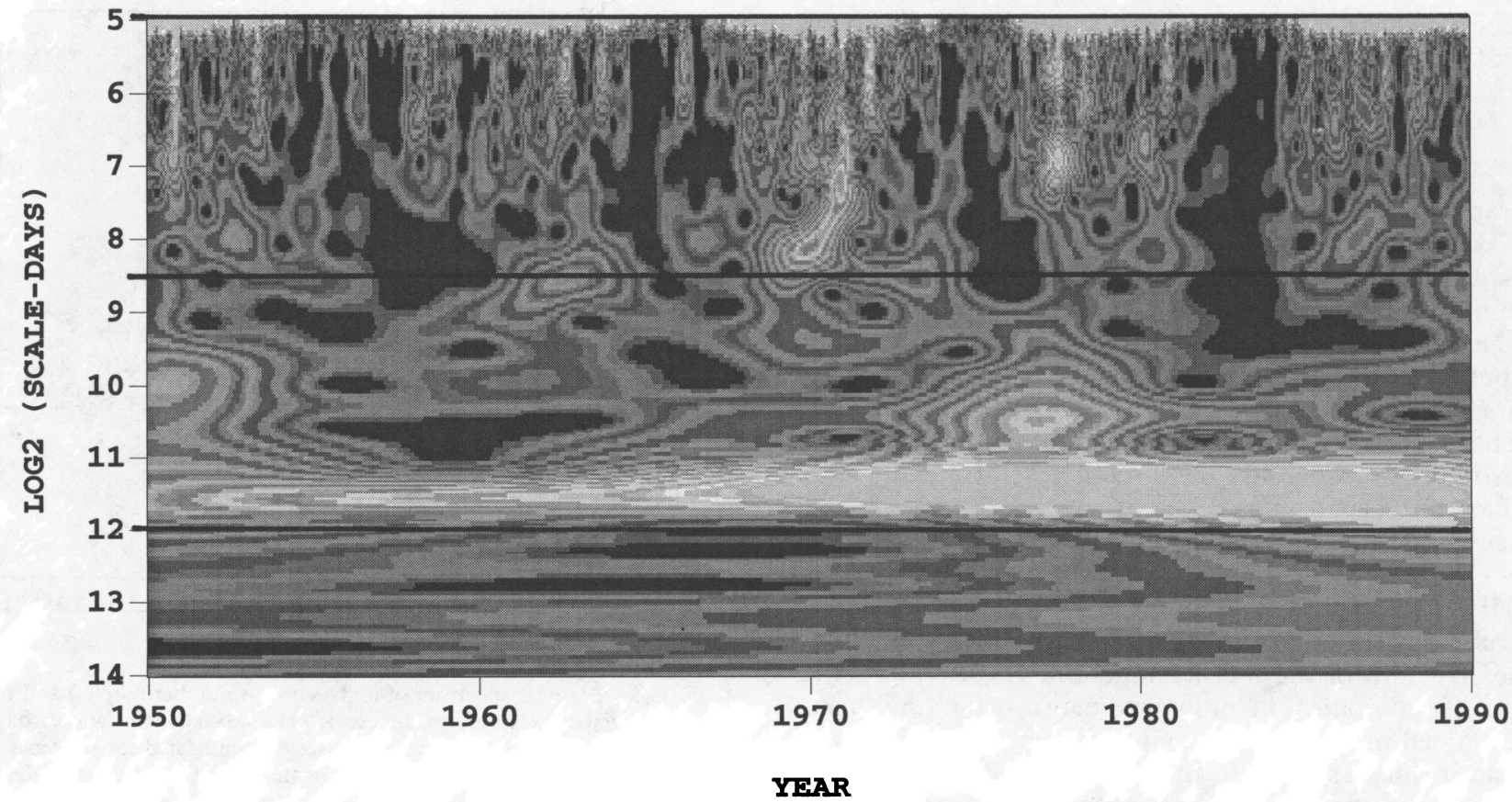

Fig. 5.-Modulus of the Morlet wavelet transform of monthly sunspot numbers from 1950 to the present. The horizontal axis indicates the dates of features. The top row shows temporal features of timescale 1 month $\approx 2^{5}$ days. Also indicated are reference lines for timescale $1 \mathrm{yr} \approx 2^{8.5}$ days and for timescale $11.2 \mathrm{yr} \approx 2^{12}$ days.

changes in the length of individual cycles: upward for shorter cycles, downward for longer ones. In the lower, long-timescale half of the plot the main features appear to be at period doubling of the $11.2 \mathrm{yr}$ cycle at $\sim 22 \mathrm{yr}, \sim 45 \mathrm{yr}$, and $\sim 90 \mathrm{yr}$. The $\sim 90 \mathrm{yr}$ feature extends across the image and represents the so-called Gleissberg cycle (Gleissberg 1966; Feynman \& Gabriel 1990).

\subsection{Random Multiplicative Models}

Let us focus first on the variability of the solar activity on timescales less than a year or two. Our goal is to relate the complex structures on such scales to the turbulent dynamics of the solar convection zone. We will demonstrate the turbulent character of the short-term dynamics by the use of multifractal measures.

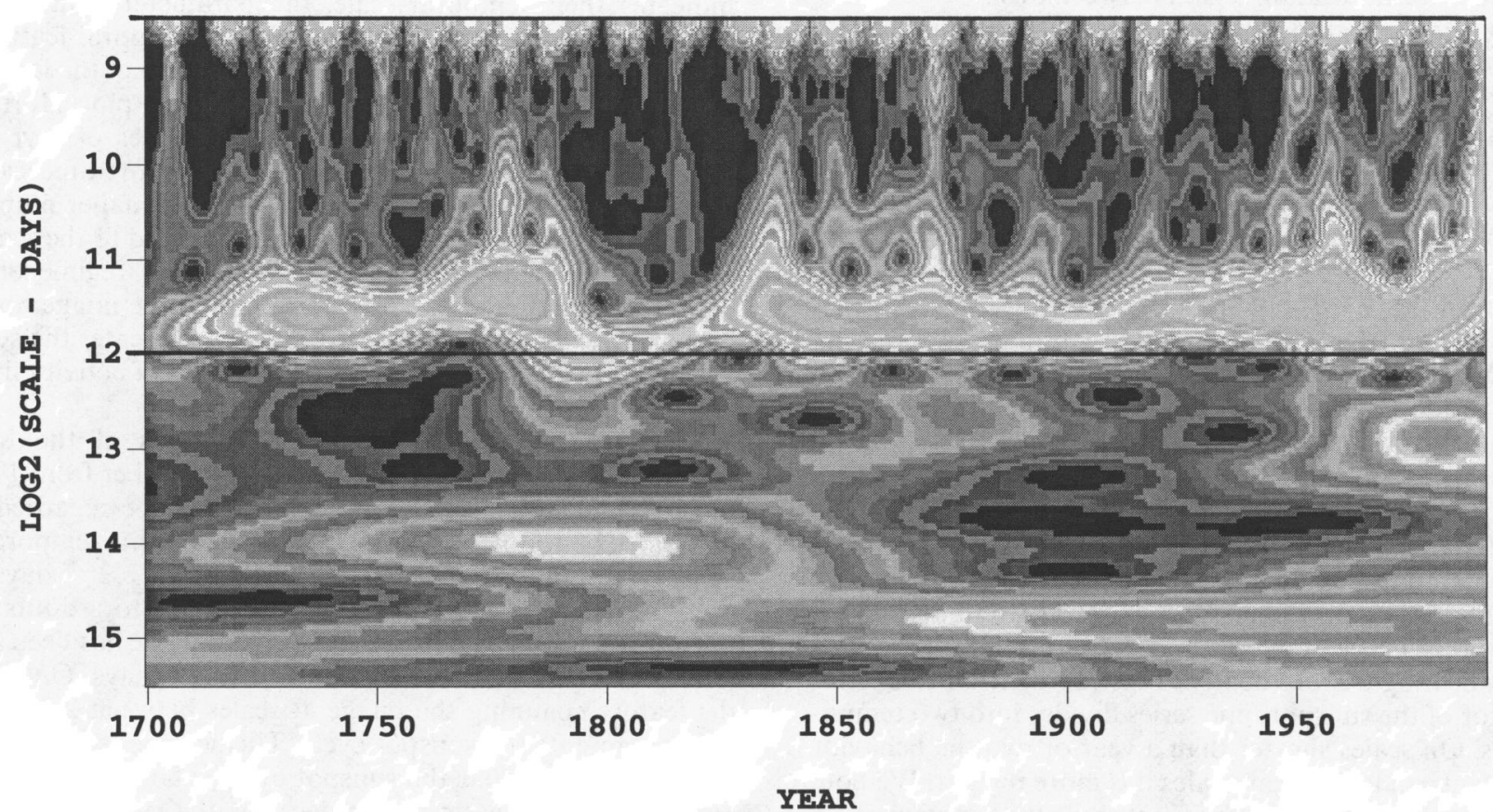

Fig. 6.-Modulus of the Morlet wavelet transform of yearly sunspot numbers from 1700 to the present. The horizontal axis indicates the dates of features. The top row shows temporal features of timescale $1 \mathrm{yr} \approx 2^{8.5}$ days. Also indicated is a reference line for timescale $11.2 \mathrm{yr} \approx 2^{12}$ days. 


\subsubsection{Measure Constructed from a Multiplicative Cascade}

Multifractal measures typically are constructed as follows. A uniform measure on, say, the unit interval is divided between the two halves of the interval, either according to a fixed multiplier (say, $M_{0}$ and $1-M_{0}$ ) or according to a multiplier selected at random from a known probability distribution $P(M)$, where conservation of the measure requires that $P(M)=P(1-M)$. The deterministic case, of course, corresponds to a delta function probability density: $P(M)=$ $0.5\left[\delta\left(M-M_{0}\right)+\delta\left(M-1+M_{0}\right)\right]$. Next, the measure contained in the subsets is divided again among sub-subsets according to the same rule. Then the measure is again divided among sub-subsubsets, and so on.

The invariance of the allocation rule through all the levels of subdivision produces multifractal scaling. We can compute the multifractal spectrum $f(\alpha)$ from the moments $\left\langle M^{q}\right\rangle$ of $P(M)$, where $q$ is a continuous parameter (Chhabra \& Sreenivasan 1991a, b, 1992). In particular the singularity strength

$$
\langle\alpha(q)\rangle=-\frac{\left\langle M^{q} \log (M)\right\rangle}{\left\langle M^{q}\right\rangle \log (2)}
$$

and the multifractal spectrum

$$
f(q)=q\langle\alpha(q)\rangle+D_{0}+\frac{\log \left(\left\langle M^{q}\right\rangle\right)}{\log (2)},
$$

where $D_{0}$ is the fractal dimension of the support of the measure and the brackets denote averaging over the distribution density $P(M)$.

The multifractal spectrum $f(\alpha)$ is relatively insensitive to details of the cascade multiplier distribution density $P(M)$. In principle, this probability distribution can be used to calculate the full multifractal spectrum, but the reverse is not true (Chhabra, Jensen, \& Sreenivasan 1989). Thus $P(M)$ is the more fundamental quantity. It has the further advantage of taking only finite values on a finite range. This in turn makes its interpretation in terms of image characteristics more transparent. For example, it is easy to display the degree of scale invariance that may be present. The probability distribution also is more closely related to the actual physics of the cascade process by which the observed field distributions are presumed to be built up.

\subsubsection{Finding a Multiplicative Process from Data}

We estimate the scale-invariant probability distribution $P(M)$ from our time series as follows (Chhabra \& Sreenivasan 1991a, b, 1992). First we choose a scale $s=2 n$ days. We then locate an interval of this length at random in the data, divide this into halves of side $n$, and determine the ratio of the measure in each to the sum. This gives a sample value of $M$. The choice of interval is repeated many times to build up a distribution. Then a new scale is selected and a new distribution built up, and so on. Symmetry of $P(M)$ about $M=0.5$ is forced by the assumed conservative nature of the cascade process. If the image being analyzed is completely uniform, then the measure will always be divided equally between adjacent squares, and we will have $M=0.5$ in every case. Then $P(M)=\delta\left(M-\frac{1}{2}\right)$. Thus a narrow distribution, peaked about $M=0.5$, indicates a relatively uniform measure. An intermittent measure will have a broader $P(M)$.

Figure 7 is a surface plot of the multiplier distribution density $P(M)$ for a wide range of "coarse-graining" timescales $s$ from 2 to 4096 days. As mentioned above, this is analogous to a Haar wavelet spectrum, the output of which at each scale is

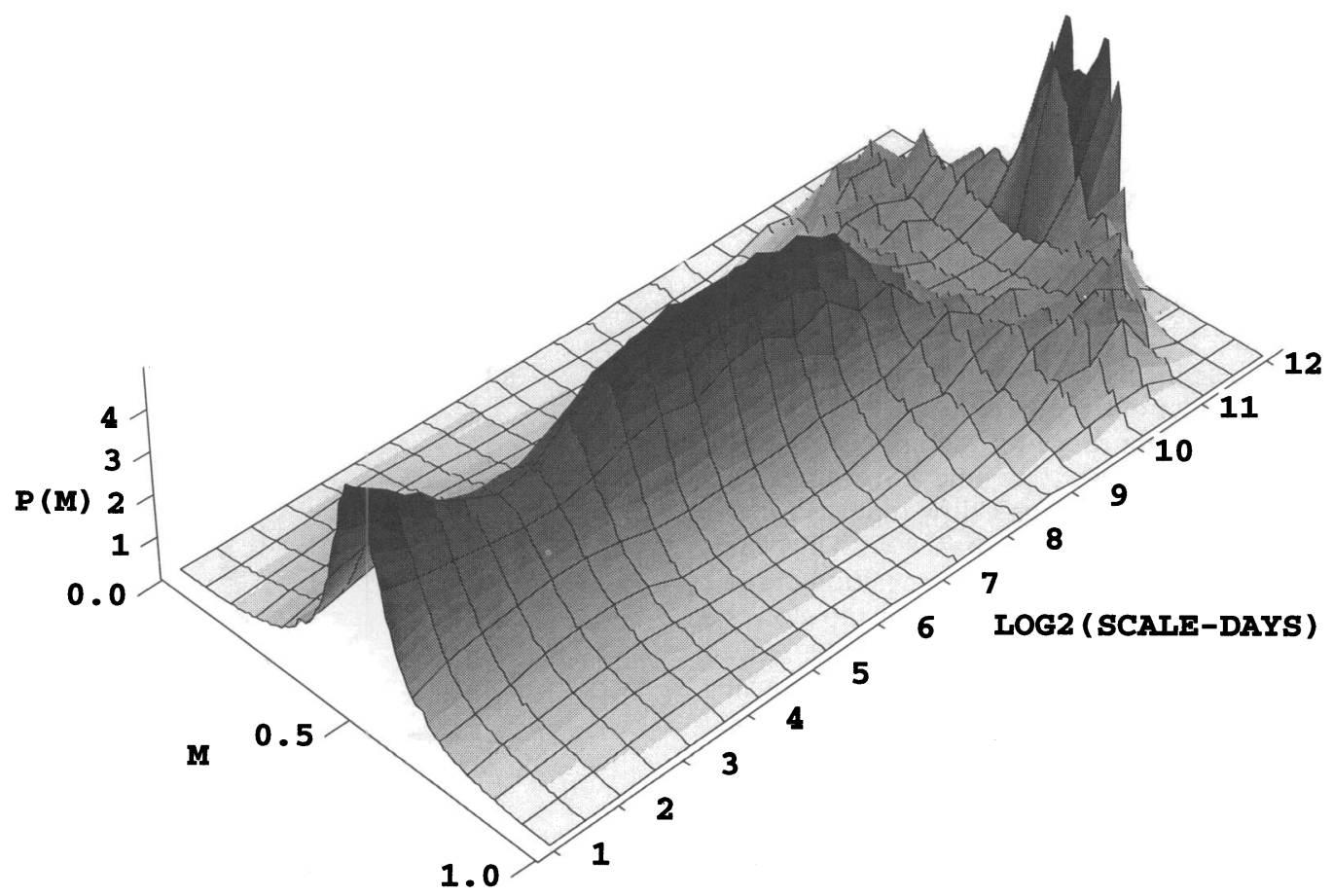

Fig. 7.-Multiplier probability distribution density $P(M)$ vs. multiplier $M$ for sunspot number for timescales from $2^{1}$ days to $2^{12}$ days $\approx 11.2$ yr. Note that the solar equatorial rotation period is $2^{4.75} \approx 27$ days and that $2^{8.5}$ days $\approx 1 \mathrm{yr}$. 
the probability distribution density $P(M)$ rather than a single number. At a timescale of 2 days the $P(M)$ is highly peaked near $M=0.5$. This reflects the fact that the sunspot number varies continuously over a few days time. At longer time intervals $P(M)$ becomes broader and lower (it is always normalized to unity) through a saddle point with minimum at an interval approximating half the solar rotation period. At this timescale, the adjacent intervals being compared incorporate the activity in opposite solar hemispheres. Since the activity is not uniformly distributed in solar longitude the $P(M)$ is broadened.

Time intervals greater than $\sim 27$ days each cover the entire solar sphere, so the rotational signal no longer is apparent. From this scale up to $\sim 256$ days, about an order of magnitude in scale, $P(M)$ remains invariant. For longer timescales the distribution widens into a bimodal pattern with peaks at $M=0.2,0.8$ at time intervals spanning 3-4 yr. This represents adjacent time intervals sampling different portions of the $11 \mathrm{yr}$ sunspot cycle. At a time interval $2^{12} \approx 4096$ days $\approx 11 \mathrm{yr}$ the time intervals sample an entire cycle, so this signal is no longer apparent. At still longer timescales, $P(M)$ remains narrow, but with variable sharp peaks, symmetrically placed about $M=0.5$. This may be characteristic of the temporal evolution of a dynamical system with a low-dimensional attractor. Indeed, a random multiplier analysis of time series generated by the well-known Lorentz (1963) system produce results resembling those of the long-timescale end of Figure 7.

The range over which the multiplier probability density is seen in Figure 7 to be scale invariant is bounded below by the solar rotation and above by effects of the sunspot cycle. We argue, however, that this range can be extended in both directions.

The effect of the solar rotation reflects only the heliocentric longitude of Earth, not the condition of the photospheric fields. Thus the range of scale invariance of $P(M)$ should extend down to time intervals as short as 2 or 3 days. This may extend to even shorter timescales. The apparent continuity of the sunspot number from day to day may represent the minimum lifetime of spots large enough to be visually detected and recorded. Certainly days with sunspot number zero do not represent a total absence of surface magnetism. Because of this, in calculating the $P(M)$ we have dropped those cases with $M=0,1$. When these points are included, they add delta function peaks to $P(M)$ at $M=0,1$ for scales up to about a solar rotation. These delta functions are disjoint from the rest of the distribution density and do not appear to characterize the physics of the system. Their inclusion or exclusion has no significant effect on the remainder of the distribution.

The interruption of the scaling range on the upper end represents a true change in the physical regime governing the variation of solar activity. We shall argue below that the longscale regime operates for intervals longer than $8 \mathrm{yr}$. Thus the $P(M)$ at timescales of 1 or 2 yr represents a superposition of the shorter time, scale-invariant variability pattern on the slower solar cycle. By applying a high-pass Fourier filter to the daily sunspot time series, it is possible to extend the upper limit of the range over which $P(M)$ is scale invariant up to $\sim 2 \mathrm{yr}$. Figure 8 displays the shape of $P(M)$ for timescales 3 days to 724 days, including one plot at scale 11 days showing the broadening due to solar rotation. On the basis of the above we argue for a $\sim 2.5$ decade range of scale invariance of the multiplier probability distribution density extending from $\lesssim 2$ days to $\sim 2$ yr. As a check on this fundamental result, Figure 9 shows the same results for $F_{10.7}$. Here again the data have been pro-

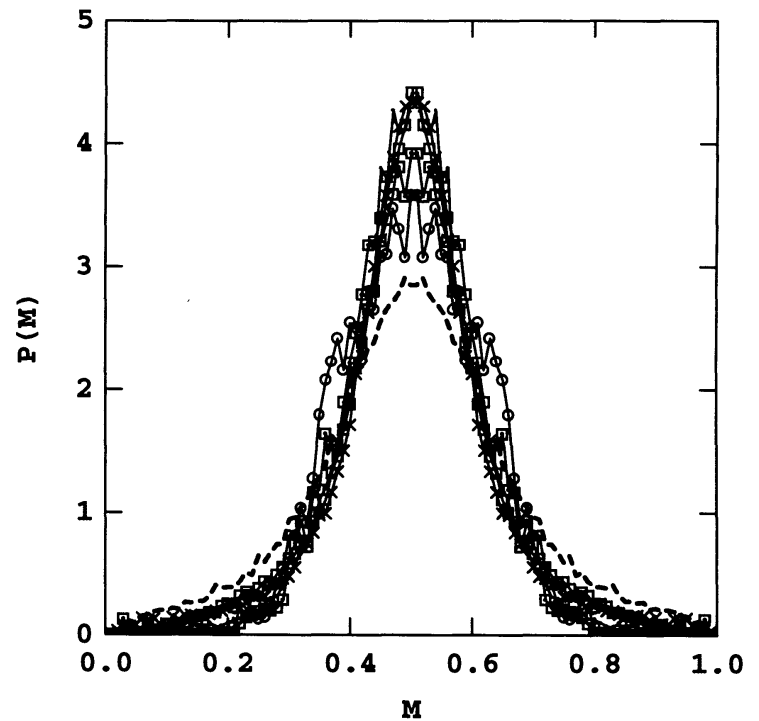

FIG. 8.-Multiplier probability distribution density $P(M)$ vs. multiplier $M$ for high-pass filtered sunspot number for timescales 3 days (squares), 11 days (dashed line), 32 days (crosses), 128 days (dots), 362 days (dotted squares), and 724 days (circles). The dashed-line curve at timescale 11 days shows broadening due to the solar rotation period.

cessed with a high-pass filter to remove long-term structure. The distribution density is narrower than that for sunspot number. Once again, however, $\sim 2.5$ decades of scale invariance are apparent. The same results also can be found for the other data sets listed in $\S 2$ above, to an accuracy commensurate with an order-of-magnitude fewer data points.

\subsubsection{Turbulent Character of the Short-Term Dynamics}

Thus in the range from about 2 days to $2 \mathrm{yr}$ we see evidence for a high-dimensional, stochastic interpretation of the variability, as opposed to a low-dimensional, chaotic interpretation.

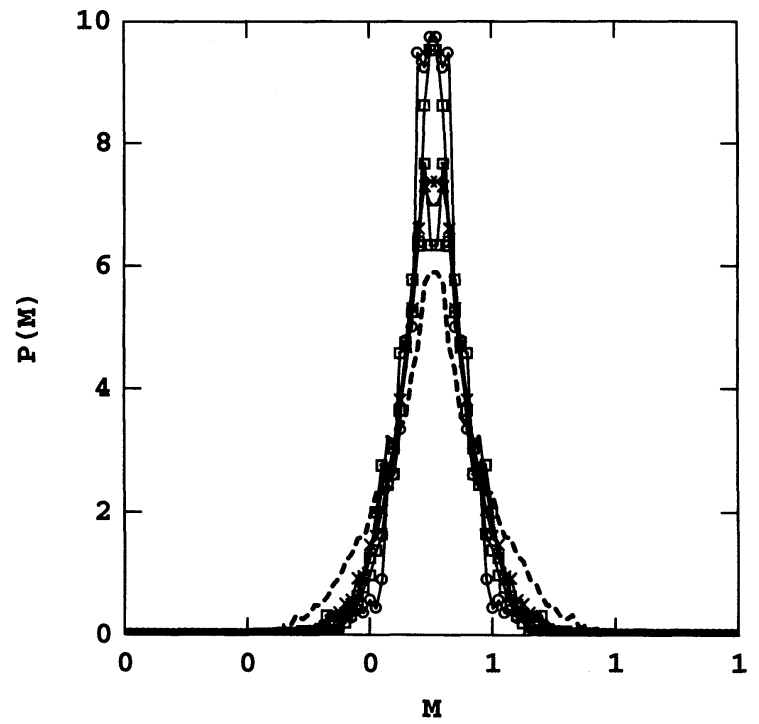

FIG. 9.-Multiplier probability distribution density $P(M)$ vs. multiplier $M$ for high-pass filtered $F_{10.7}$ for timescales 3 days (squares), 11 days (dashed line), 32 days (crosses), 128 days (dots), 362 days (dotted squares), and 724 days (circles). The dashed-line curve at timescale 11 days shows broadening due to the solar rotation period. 


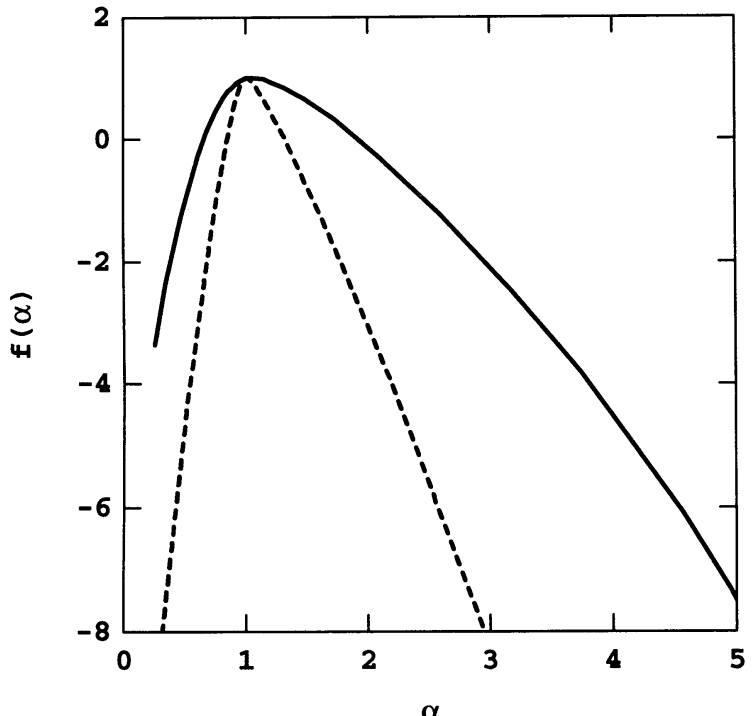

FIG. 10.-Multifractal spectrum $f(\alpha)$ vs. singularity strength $\alpha$ for the daily sunspot number (solid line) and $F_{10.7}($ dashed line)

Such high-dimensional dynamics are very intermittent. Figure 10 shows the multifractal singularity spectra $f(\alpha)$ calculated from $P(M)$ for the daily sunspot numbers and the $F_{10.7}$ flux using equations (3.6) and (3.7). The result for the microwave flux (dotted line) is much narrower, reflecting the fact that the $F_{10.7}$ curve in Figure 1 shows a nearly one-dimensional measure. In the exact one-dimensional case the corresponding multifractal spectrum would collapse to a single point at $f=1$, $\alpha=1$. Both multifractal spectra, however, extend below $\alpha=1$, indicating the presence of singular peaks (i.e., intermittency) in both solar activity measures. This means that throughout the range of self-similar scaling, however, far down it may extend, there are peaks whose density (measure per unit time) continues to increase when viewed at finer and finer resolution.

The spectra in Figure 10 are derived from the moments of the multiplier probability densities $P(M)$ by using $q$ as a free parameter in equations (3.6) and (3.7) and finding the corresponding $f$ and $\alpha$. Implicit in this computation is the scale invariance of the $P(M)$ which we have discovered. Of special interest is the case $q=2$. It was found (Chhabra \& Sreenivasan 1992) that for different realizations of high-dimensional, stochastic cascade processes, the values of $f(2)$ and $\alpha(2)$ are variable. Further, the different points $f(2)$ and $\alpha(2)$ fall along a straight line as shown in Figure 11. We show in this figure data points taken from the Chhabra \& Sreenivasan paper representing 10 stochastic numerical cascade simulations as well as 10 time series of the dissipation measure in a terrestrial atmospheric boundary layer. We have added to this plot points corresponding to the distribution of magnetic fields in five high-resolution solar magnetic images (Lawrence et al. 1995). Here $f$ and $\alpha$ have been divided by 2 because they were derived from two-dimensional data sets. The data points fall on the same straight line as the simulations and terrestrial data. In addition, we have included data points derived from a number of time series representing solar activity. These were the daily and monthly sunspot numbers (whose data points effectively coincide), the daily Photometric Sunspot Index (Wilson et al. 1981) daily Nimbus 7 solar irradiance variations (Hoyt et al. 1992), the daily Standford mean magnetic flux and daily

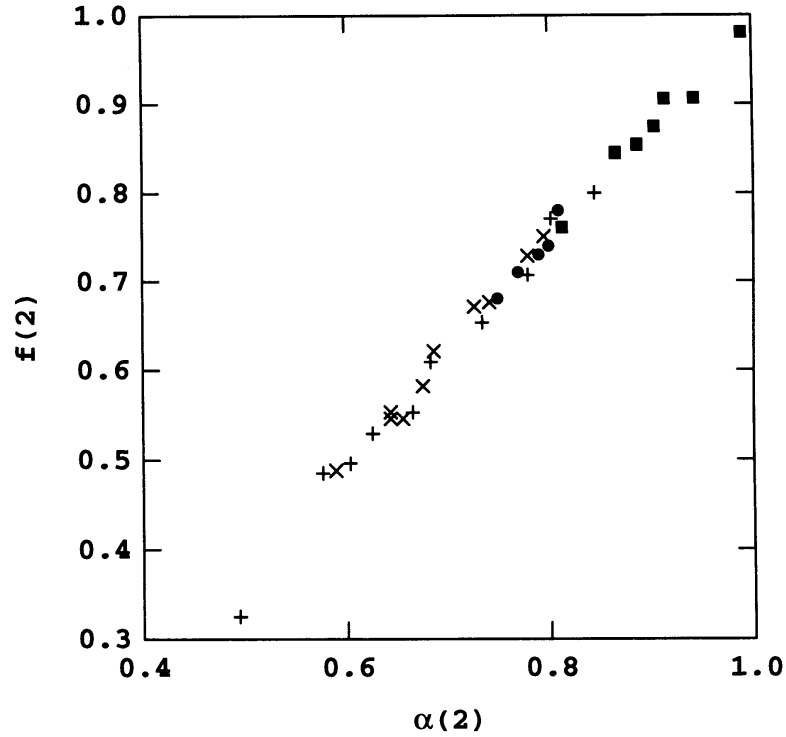

Fig. 11.-Multifractal spectrum $f(2)$ vs. singularity strength $\alpha(2)$ from the $q=2$ moment of $P(M)$ for 10 generated measures (plus signs) and for 10 samples of a terrestrial atmospheric boundary layer (crosses) from Chhabra \& Sreenivasan (1992). Also included are spatial measures in five solar magnetic images (circles) and for eight solar magnetic time series (squares).

calcium emission index from NOAA's World Data Center, and ${ }^{14} \mathrm{C}$ fluctuations at $20 \mathrm{yr}$ intervals from 6000 B.C. to the present (Suess 1978). All of these give points falling on the same straight line in Figure 11. This indicates that widely diverse turbulent systems, including the solar convection zone, share "universal" aspects of behavior.

This interpretation differs from the suggestion that shortterm fluctuations in the sunspot number may represent a lowdimensional Lorentz dynamical system (Morfill et al. 1991). Further, we find in neither the correlation function nor the mutual information an indication of a characteristic 50 day correlation time associated with this suggestion.

\subsection{Chaotic Attractor}

Now we turn to the long-term dynamics. Mundt et al. (1991), have found evidence for the existence of low-dimensional, $D=2.3$, chaos in the monthly sunspot number series after lowpass filtering to remove fluctuations on timescales $<6 \mathrm{yr}$. Our aim here is to extend the method of Grassberger \& Proccacia (1983a) to establish a lower limit timescale for the operation of this low-dimensional, chaotic system.

The evolution of a low-dimensional, chaotic system is presumed to be described by a small number $n$ of coupled, nonlinear, first-order differential equations. In the case of the Lorentz system, $n=3$. The system evolution is trajectory in an $n$ dimensional phase space. Typically, the trajectory winds on itself in a bounded volume of the phase space of linear size $L$. The defining characteristic of chaos is that neighboring, and nearly parallel, segments of the trajectory diverge exponentially up to a separation of order $L$. The points of space occupied by the trajectory (the "attractor") typically have a fractal correlation dimension $D_{2}<n$. For the Lorentz system, $D_{2}=2.06$. (Actually, because the trajectory visits different portions of the attractor with different frequency, it generates a multifractal measure with generalized dimensions $D_{q}$.)

In practice, we are given a time series, like the sunspot number, and must try to deduce the presence and properties of 
the attractor therefrom. We employ the method of Grassberger \& Procaccia (1983a). If one envisions a finite-difference version of the $n$ coupled differential equations governing the system, it is apparent that the information contained in the $n$ independent variables also is contained in $n$ equally spaced values of any one of the variables separated by an arbitrary time interval $\tau$. We do not know $n$ to begin with, so we embed the trajectory in a Euclidean space of variable trial dimension $d$. The points of the trajectory are represented by $d$-dimensional vectors $\boldsymbol{X}_{i}=\left\{x\left(t_{i}\right), x\left(t_{i}+\tau\right), x\left(t_{i}+2 \tau\right) \ldots x\left[t_{i}+(d-1) \tau\right]\right\}$. The interval $\tau$ is in principle arbitrary. As a practical matter, it cannot be too small or the continuous trajectory will appear onedimensional. If $\tau$ is so large than the trajectory traverses the volume of size $L$, then the components of the vector will be completely uncorrelated. We have used a 13 day interval based on the first minimum of the mutual information (Fig. 2). Mundt et al. (1991) used $\tau=10$ months to find similar results. This verifies that the result is only weakly, if at all, dependent on $\tau$.

From the cloud of data points in the $d$-dimensional Euclidean space, we generate the correlation integral

$$
C(r)=\lim _{N \rightarrow \infty} \sum_{i, j=1}^{N} \Theta\left(r-\left\|X_{i}-X_{j}\right\|\right),
$$

where $\left\|\boldsymbol{X}_{i}-\boldsymbol{X}_{j}\right\|$ notation refers to the $d$-dimensional Euclidean distance between two points. $C(r)$ is proportional to the number of pairs of data points located within a $d$-dimensional Euclidean distance $r$ of one another. If, over some range of $r$,

$$
C(r) \propto r^{D_{2}}
$$

the attractor has a correlation dimension $D_{2}$ (the subscript refers to the correlation of pairs of points). If the trial dimension $d$ is chosen too small, then many pairs of data points will appear falsely to be neighbors by projection, in the sense that the shadows of separated objects may lie close together, and the value obtained for $D_{2}$ will be incorrect. As the trial embedding dimension $d$ is increased, the measured $D_{2}$ will increase. Eventually, $d$ will be large enough $(d>2 n+1$ is the sufficient condition) that all projection effects are removed, and the correct $D_{2}$ will be obtained. Further increases of $d$ produce no further increase in $D_{2}$. For truly random points, however, $D_{2}$ is infinite and will grow endlessly with increasing $d$.

Mundt et al. (1991) have carried out this analysis for the monthly sunspot number $1800-1991$, filtered to remove fluctuations more rapid than $\sim 6 \mathrm{yr}$, with $\tau=10$ months, and found an attractor dimension $D_{2} \approx 2.3$. For the unfiltered daily sunspot number $1818-1991$ we find $D_{2} \approx 2.0-2.3$ for $d \approx$ $10-40$ in good agreement (see Fig. 12). Each of these analyses is imperfect for a different reason. The monthly numbers, on the one hand, provided about 2300 data points, only 1000 per attractor dimension. The daily numbers, on the other hand, provide over 27,000 points per attractor dimension but are contaminated to some extent by the solar rotation. Nevertheless, both approaches indicate the presence of an attractor, and they agree on its dimension. The result, therefore, appears quite robust.

Gizzatullina et al. (1990) have estimated an attractor dimension $D_{2} \approx 3.3$ for deviations in the ${ }^{14} \mathrm{C}$ record from 6000 B.C. to the present. This is consistent with the sunspot result given the presence of an additional degree of freedom. By the same procedure, Kürths \& Herzel (1989) have found indications of a low-dimensional attractor in radio emissions from a solar flare.

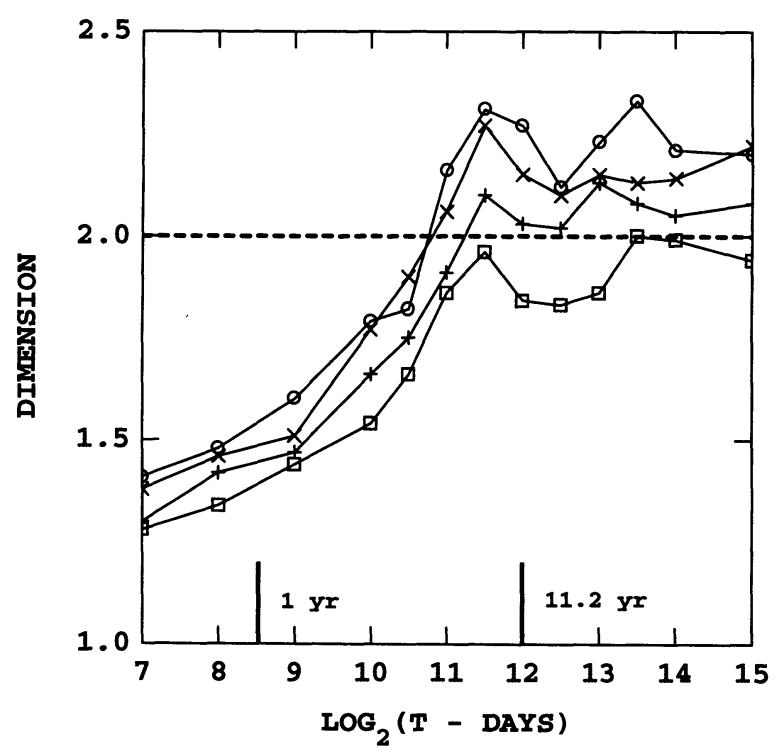

Fig. 12.-Attractor dimension for sunspot number time series vs. time horizon $T$ in days. The dashed line indicates $D=2$. Also indicated are reference marks for timescale $1 \mathrm{yr} \approx 2^{8.5}$ days and for timescale $11.2 \mathrm{yr} \approx 2^{12}$ days.

We have carried out a variant of this analysis to attempt to determine the timescale of transition in the daily sunspot number from short-time, stochastic (or high-dimensional chaotic) behavior to low-dimensional chaos at longer times. To do this, when we calculate the correlation integral $C(r)$, for $d \gg 2 n+1$, we include only pairs of data points with temporal separation less than a time horizon $T$. For unrestricted timing, the neighborhood of a given point will include other points from several different loops of the system's trajectory in phase space, and the global properties of the attractor will determine the resulting dimension. When a time horizon $T$ is applied, and when $T$ is reduced, fewer and fewer such loops will be included, and eventually only one. In this situation, the short-timescale physics will dominate, We find that when $T \lesssim 8$ yr the measured $D_{2}$ varies directly with $T$, but for $T \gtrsim 8 \mathrm{yr}$ it maintains a constant value $D_{2} \approx 2.0-2.3$. This is shown for $d=10,20,30$, 40 in Figure 12. Thus the low-dimensional attractor appears to operate entirely above a timescale of $8 \mathrm{yr}$. At shorter timescales, the dominant process appears to be high-dimensional or stochastic. The abruptness of the saturation, together with the fact that for $T<8 \mathrm{yr}$ the data converge well to a reduced attractor dimension, indicates that this is not merely the effect of poor statistics resulting from truncation of the sample of attractor points.

This result has been checked further by computation of the Kolmogorov-Sinai entropy for the sunspot attractor using the method of Grassberger \& Procaccia (1983b). This is equivalent to the sum of the positive Lyapounov exponents of the system. We find that for $T \gtrsim 8$ this correctly converges to a finite value but fails to do so for $T \lesssim 8 \mathrm{yr}$.

\section{DISCUSSION AND CONCLUSIONS}

We have distinguished two different timescale regimes in solar magnetic variability. As had previously been expected (Ruzmaikin 1981; Weiss et al. 1984), Mundt, Maguire, \& Chase (1991) have discovered a low-dimensional, chaotic behavior in the sunspot number. We have found that this operates at timescales longer than a transition threshold scale of 
about 8 yr. Second, at timescales below about 2 yr the variability can be characterized by a random multiplicative cascade process that is invariant over two decades of scale. This indicates turbulent structuring of the solar fields as they rise through the convection zone.

Some quantitative estimates help to put this picture into perspective. The turbulent diffusivity of the plasma in the convection zone is thought to be $\eta_{T} \approx 5 \times 10^{12} \mathrm{~cm}^{2} \mathrm{~s}^{-1}$, and the depth of the convection zone is $h_{c z} \approx 2 \times 10^{10} \mathrm{~cm}$. Thus, the diffusion time from the bottom of the convection zone to the photosphere would be $\tau \approx h_{c z}^{2} \eta_{T}^{-1} \approx 3$ yr. This number is comparable to, but longer than, the timescale of the longest cascade structures discussed ( $\lesssim 2 \mathrm{yr}$ ). It also is comparable to, but shorter than, the timescale range ( $\gtrsim 8 \mathrm{yr})$ at which we have found the low-dimensional dynamical system to operate.

The multiplicative cascade process we have put forward involves structuring of the data with correlations on all scales. It is not difficult to understand how this could work for spatial data, such as the distribution of fields in active regions. Questions of causality complicate the interpretation of temporal data. Often, however, apparently temporal data actually are spatial data sampled sequentially. We suggest such an interpretation here. The magnetic structures leading to sunspots rise to the solar surface from within the convection zone through turbulent plasma motions. In this process the fields are spatially structured by the turbulence and then sequentially sampled upon arrival at the photosphere.

Our picture of the spatial structuring of magnetic fields in the convection zone is supported by direct, numerical simulations of hydromagnetic turbulence (Brandenburg et al. 1992). This simulation was designed to correspond to conditions in the solar covection zone, although the Reynolds number value ( 300) was much lower. The magnitude of the magnetic field vector $B=|B|$ was found to scale multifractally by the method of generalized dimensions. A one-dimensional sampling of this distribution would produce a spectrum of the form of that in Figure 10.

The hypothesis that generic turbulence structures solar magnetic activity is based on the notion of "universality" in physics: that many physical systems, in diverse areas of physics, operate according to some common abstract principle. The "universality class" containing both terrestrial atmospheric turbulence and the sunspot fluctuations is defined, first, by a general scale invariance, and, second, by a the presence of a scale-invariant multiplicative cascade process with multiplier probability distribution density $P(M)$ with the characteristic shape of Figures 7, 8, and 9. This second criterion excludes such intermittent and scale-invariant processes as colored noise and low-dimensional chaos.

A drawback to our viewpoint is that the mere discovery of universal, or generic, behavior provides no further insight into the particular mechanism of the physical system at hand, in the present case magnetohydrodynamic turbulence. Our goal here has been only to establish the turbulent nature of the suspot time series. An advantage for the future, however, is that one may try to understand some aspects of the behavior of a complicated system by studying the properties of much simplified "toy" systems belonging to the same universality class. We hope, in future work, to exploit this advantage in modeling of short-time, small-scale solar dynamo mechanisms. The longtime, large-scale mechanisms seem best modeled by lowdimensional dynamical systems. Magnetic variability on scales between $2 \mathrm{yr}$ and $8 \mathrm{yr}$ appear to be analytically intractable and to require handling by direct simulation.

A. C. C. acknowledges released time provided by the CSUN Research and Grants Committee. This work was supported in part by NSF grants ATM 91-15111 and ATM 93-09901.

\section{REFERENCES}

Abarbanel, H. D. I., Brown, R., Sidorowich, J. J., \& Tsimring, L. Sh. 1993, Rev. Mod. Phys., 65, 1331

Brandenburg, A., Procaccia, I., Segel, D., \& Vincent, A. 1992, Phys. Rev. A, 46, 4819

Bray, R. J., \& Loughhead, R. E. 1964, Sunspots (London: Chapman \& Hall), 237

Cadavid, A. C., Lawrence, J. K., Ruzmaikin, A. A., \& Kayleng-Knight, A. 1994 ApJ, 429, 391

Chhabra, A. B., Jensen, C., \& Sreenivasan, K. R. 1989, Phys. Rev. A, 40, 4953

Chhabra, A. B., \& Sreenivasan, K. R. 1991a, Phys. Rev. A, 43, 1114

. 1991b, in New Perspectives in Turbulence, ed. L. Sirovich (Berlin, Springer), 271

1992 Phys. Rev. Lett 68, 2762

Farge, M. 1992, Ann. Rev. Fluid Mech., 24, 395

Feynman, J., \& Gabriel, S. B. 1990, Sol. Phys., 127, 393

Gallager, R. G. 1968, Information Theory and Reliable Communication (New York: Wiley)

Gizzatullina, S. M., Rukavishnikov, V. D., Ruzmaikin, A. A., \& Tavastsherna, K. S. 1990, Sol. Phys., 127, 281

Gleissberg, W. 1966, J. British Astron. Assoc., 76, 265

Grassberger, P., \& Procaccia, I. 1983a, Phys. Rev. Lett., 50, 346
Grassberger, P., \& Procaccia, I. 1983b, Phys. Rev. A, 28, 25

Hoyt, D. V., Kyle, H. L., Hickey, J. R., \& Maschhoff, R. H. 1992, J. Geophys Res., 97,51

Kürths, J., \& Herzel, H. 1989, Physica D, 25, 165

Lawrence, J. K., Cadavid, A. C., \& Ruzmaikin, A. A. 1994, in Solar Surface Magnetism, ed. R. J. Rutten \& C. J. Schrijver (Dordrecht: Kluwer), 279 1995, Phys. Rev. E, 51, 316

Lawrence, J. K., Ruzmaikin, A. A., \& Cadavid, A. C. 1993, ApJ, 417, 805

Lorentz, E. N. 1963, J. Atmos. Sci., 20, 130

Meneveau, C. 1991, J. Fluid Mech., 232, 469

Morfill, G. E. Scheingraber, H., Voges, W., \& Sonett, C. P. 1991, in The Sun in Time, ed. C. P. Sonett, M. S. Giampapa, \& M. S. Matthews (Tucson: Univ. of Arizona Press), 30

Mundt, M. D., Maguire, W. B., II, \& Chase, R. R. P. 1991, J. Geophys. Res., 96, 1705

Ruzmaikin, A. A. 1981, Comments Astrophys., 9, 85

Suess, H. E. 1978, Radiocarbon, 20,1

Weiss, N. O., Cattaneo, F., \& Jones, C. A. 1984, Geophys. Astrophys. Fluid Dyn., 30,305

Willson, R. C., Gulkis, S., Janssen, M., Hudson, H. S., \& Chapman, G. A. 1981, Science, 234,1114 